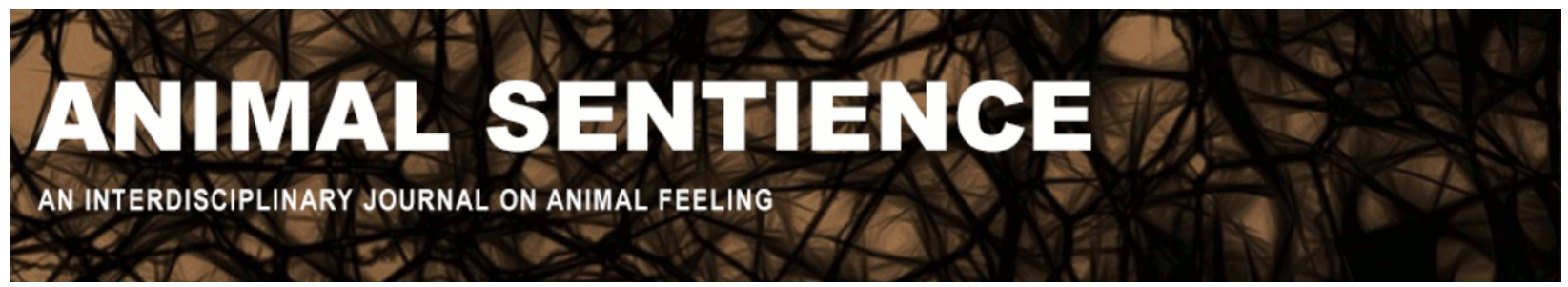

Karl, Sabrina and Huber, Ludwig (2017) Empathy in dogs: With a little help from a friend - a mixed blessing. Animal Sentience 14(13)

DOI: $10.51291 / 2377-7478.1271$

Date of submission: 2017-12-04

Date of acceptance: 2017-12-06 (c) 


\title{
Empathy in dogs: With a little help from a friend - a mixed blessing
}

Commentary on Kujala on Canine Emotions

\author{
Sabrina Karl \\ Comparative Cognition, Messerli Research Institute \\ University of Veterinary Medicine Vienna \\ Ludwig Huber \\ Comparative Cognition, Messerli Research Institute \\ University of Veterinary Medicine Vienna \\ Medical University of Vienna \& University of Vienna
}

\begin{abstract}
Kujala (2017) presents an extensive overview of existing research on canine emotions in comparison to those of other non-human animals and humans. This commentary provides some additional research results on the intensively debated field of empathy in dogs. We focus on recent advances in the understanding of a fundamental building block of empathy - emotional contagion - and on dogs' remarkable sensitivity for human emotions, including the skills of assistance dogs.
\end{abstract}

Sabrina Karl is a PhD student at the University of Veterinary Medicine Vienna. Her PhD project is about the human-dogattachment system including behavioral studies and functional brain imaging in dogs.

www.vetmeduni.ac.at/en/messerli/scienc e/cognition/staff/phd-candidates/sabrinakarl/

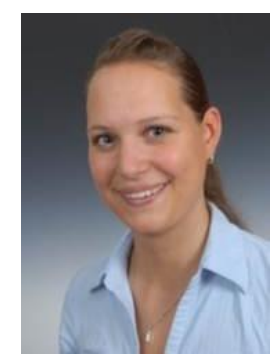

\begin{abstract}
Ludwig Huber co-founded the Department of Cognitive Biology at University of Vienna, where he is professor of animal ethics and human-animal interactions. His research involves social learning, imitation, and empathy in dogs and marmosets. He is Head of the Comparative Cognition Unit and Clever Dog Lab at the Haidlhof Research Station. www.vetmeduni.ac.at/en/messerli/science/cognition/staff/staf f/ludwig-huber/
\end{abstract}

1. Empathy in dogs. To study empathy and emotional contagion (Preston \& de Waal 2002; Custance \& Mayer 2012), Huber and colleagues (2017) tested dogs' behavioral responses to different recorded sounds. They presented positive (laugh/play bark) and negative (cry/isolation whine) sounds. The sounds came not just from conspecifics, but also from humans, with various non-emotional (abiotic/biotic) sounds as the control. As expected, the dogs had different behavioral reactions to these sounds. With emotional sounds, they were more attentive and showed more behavioral signs of emotional states. Emotional sounds from conspecifics - whether positive or negative - caused more freezing than human emotional sounds. There was arousal after hearing negative emotional sounds; this indicated 
emotional contagion for negative sounds as well as the ability to distinguish between different positive and negative emotions. Together with other findings from our lab (Quervel-Chaumette et al. 2016), these results provide further evidence of emotional contagion for negative sounds from humans and conspecifics.

Similar effects of negative emotional stimuli have recently been reported in the visual domain. Dogs performed more mouth-licking behavior when they had viewed pictures of negative emotional facial expressions from humans and conspecifics (Albuquerque et al. 2018). Interestingly, the frequency of mouth-licking behavior was higher for human than conspecific faces. Since mouth-licking behavior is mostly associated with reactions to stress, this might indicate a functional response. However, emotions are difficult to determine and there is no valid measure yet, especially for the observer's affective states. The difficulty is to distinguish between positive and negative states if the expressions are similar or too subtle. Stress-related behaviors are relatively easy to identify, but how can we discern subtle behavioral responses to positive stimuli or emotions? A facial expression ethogram for dogs does not exist, although coding tools like DogFACS (Waller et al. 2013) offer possible solutions for objective, reliable and standardized measurement of facial movements.

2. Interspecific connection. Beyond their sensitivity to intraspecific emotional context, dogs also seem to be sensitive to human emotional states (Müller et al. 2015). Custance \& Mayer (2012) found that dogs oriented more often towards human caregivers or strangers when they suddenly pretended to cry compared to when they talked or hummed. When the stranger was pretending to cry, the dogs sniffed, nuzzled and licked her instead of going to their human caregiver who was also present in the room. But why had the dogs approached the crying person more often and even stayed there when being ignored? One reason could simply be curiosity, trying to find out what the person was doing. Perhaps the dogs had never seen a person cry. The authors, however, interpreted their results, with good reason, as evidence for empathic concern towards the crying person. This raises the question of whether pretending to cry is enough to evoke the dog's empathic reaction. Dogs may recognize the difference between real and feigned crying. Macpherson \& Roberts (2006) found that when their human caregiver feigned having a heart attack or an immobilizing accident, their dogs did not go to seek help from bystander/s. It is hence quite challenging to create realistic situations to examine whether dogs really understand humans' emotions or needs.

Di Vito et al. (2010) reported that a pet dog of a patient suffering from refractory epilepsy was able to alert the patient's parents by running to them and barking at them before running back to the patient when his seizures began. At the age of one year, after having lived with the patient for eight months, he suddenly started to express special behaviors related to the patient's seizures such as barking at bystanders and later protecting the patient. There are further anecdotal reports suggesting that dogs might be able to detect seizures even before the patients themselves do. Although dogs seem to be to be able to identify real emergencies in some cases, Brown \& Strong (2001) recommended specially training dogs to perform certain behaviors in case of seizures, because dogs may also react in an anxious or aggressive way. Dogs may even try to attack the patient while they are having a seizure because they are acting differently from normal. It could also be that dogs do not understand what is happening to their human caregiver. Patients and assistance dog trainers have reported that dogs sometimes behaved as if they were in a state of high of arousal (barking, growling, anxiously running around, etc.). It is hence recommended to reinforce 
dogs when they begin to react to upcoming seizures, rewarding them for specific helping actions such as bringing a cordless phone, rolling the companion on the side to avoid aspiration, or turning off the electrical wheelchair to prevent accidents (Kirton et al. 2008). Individually trained "Seizure alert" dogs (SAD) are able to warn patients $30 \mathrm{sec}$ to $45 \mathrm{~min}$ before seizure onset (Dalziel et al. 2003). It is still not completely known how dogs detect seizures. Brown \& Goldstein (2011) have suggested that they react to subtle changes in human appearance or behavior immediately before the actual seizure starts or that they are sensitive to differences in heart rate, pheromone production or certain olfactory cues. Better experimental designs are needed in the future to clarify open questions concerning seizure dogs.

Another line of research concerns how dogs can smell different human emotional states. Human male sweat samples, taken in different emotional situations (happy, anxious), were presented to the dogs while the human caregiver, a stranger and a sweat dispenser apparatus were in the room (D'Aniolly et al. 2017). When the dogs smelled the "happy odor," they approached the stranger, but when the human odor was from a fearful situation, the dogs displayed more stressed behaviors, had higher heartrates and sought support from their human caregivers. Hence dogs' interspecies emotional communication may be based in part on chemosignals.

In conclusion, over thousands of years dogs may have developed a special relationship with humans that enabled them to communicate with us not only cognitively but also emotionally, by responding adaptively to our expressions of emotion.

\section{References}

Albuquerque, N., Guo, K., Wilkinson, A., Resende, B., Mills, D. S. (2018). Mouth-licking by dogs as a response to emotional stimuli. Behavioural Processes 146, 42-45.

Brown, S. W., Goldstein, L. H. (2011). Can seizure-alert dogs predict seizures? Epilepsy Research 97(3), 236-242.

Brown, S. W., Strong, V. (2001). The use of seizure-alert dogs. Seizure 10(1), 39-41.

Custance, D., Mayer, J. (2012). Empathic-like responding by domestic dogs (Canis familiaris) to distress in humans: An exploratory study. Animal Cognition 15(5), 851-859.

D’Aniello, B., Semin, G. R., Alterisio, A., Aria, M., Scandurra, A. (2017). Interspecies transmission of emotional information via chemosignals: From humans to dogs (Canis lupus familiaris). Animal Cognition, 1-12.

Dalziel, D. J., Uthman, B. M., McGorray, S. P., Reep, R. L. (2003). Seizure-alert dogs: A review and preliminary study. Seizure 12, 115-120.

Di Vito, L., Naldi, I., Mostacci, B., Licchetta, L., Bisulli, F., Tinuper, P. (2010). A seizure response dog: Video recording of reacting behaviour during repetitive prolonged seizures. Epileptic Disorders 12(2), 142-145.

Huber, A., Barber, A. L. A., Faragó, T., Müller, C. A., Huber, L. (2017). Investigating emotional contagion in dogs (Canis familiaris) to emotional sounds of humans and conspecifics. Animal Cognition 20(4), 703-715.

Kirton, A., Winter, A., Wirrell, E., Snead, O. C. (2008). Seizure response dogs: Evaluation of a formal training program. Epilepsy \& Behavior 13, 499-504.

Kujala, M. V. (2017). Canine emotions as seen through human social cognition. Animal Sentience 14(1). 
Macpherson, K. \& Roberts, W. A. (2006). Do dogs (Canis familiaris) seek help in an emergency? Journal of Comparative Psychology 120(2), 113-119.

Müller, C. A., Schmitt, K., Barber, A. L. A., Huber, L. (2015). Dogs can discriminate emotional expressions of human faces. Current Biology 25, 1-5.

Preston, S. D., de Waal, F. B. M. (2002). Empathy: Its ultimate and proximate bases. Behavioral and Brain Sciences 25, 1-72.

Quervel-Chaumette, M., Faerber, V., Faragó, T., Marshall-Pescini, S., Range, F. (2016). Investigating empathy-like responding to conspecifics' distress in pet dogs. PLoS One 11, e0152920.

Waller, B. M., Peirce, K., Caeiro, C. C., Scheider, L., Burrows, A. M., McCune, S., Kaminski, J. (2013). Paedomorphic facial expressions give dogs a selective advantage. PLoS One 8(12), e82686. 